\title{
The Effects of Aerobic Exercise and Gaming on Cognitive Performance
}

\author{
by \\ Peter C. Douris ${ }^{1}$, John P. Handrakis ${ }^{1}$, Demitra Apergis ${ }^{1}$, Robert B. Mangus ${ }^{1}$, \\ Rima Patel ${ }^{1}$, Jessica Limtao ${ }^{1}$, Svetlana Platonova ${ }^{1}$, Aladino Gregorio ${ }^{1}$, Elliot Luty ${ }^{1}$
}

The purpose of our study was to investigate the effects of video gaming, aerobic exercise (biking), and the combination of these two activities on the domains of cognitive performance: selective attention, processing speed, and executive functioning. The study was a randomized clinical trial with 40 subjects (mean age $23.7 \pm 1.8$ years) randomized to one of four thirty-minute conditions: video gaming, biking, simultaneous gaming and biking, and a control condition. Cognitive performance was measured pre and post condition using the Stroop test and Trails $B$ test. A mixed design was utilized. While video gaming, biking, simultaneous gaming and biking conditions improved selective attention and processing speed $(p<0.05)$, only the bike condition improved the highest order of cognitive performance, executive function $(p<0.01)$. There were no changes in cognitive performance for the control condition. Previous studies have shown that if tasks approach the limits of attentional capacity there is an increase in the overall chance for errors, known as the dual-task deficit. Simultaneous biking and gaming may have surpassed attentional capacity limits, ultimately increasing errors during the executive function tests of our cognitive performance battery. The results suggest that the fatiguing effects of a combined physically and mentally challenging task that extends after the exercise cessation may overcome the eventual beneficial cognitive effects derived from the physical exercise.

Key words: executive function, Stroop, exercise, video gaming.

\section{Introduction}

Cognitive performance $(\mathrm{CP})$ includes the processes of attention, memory, processing speed, and executive functioning. Executive function is the highest order of $\mathrm{CP}$ and is dependent on selective attention, judgment, anticipation, planning, and conflict resolution through selective inhibition occurring in the prefrontal cortex (Tam, 2013). Cognition is responsible for the selection of goal directed behaviors, which occur through the complex interaction between perception, memory, and action ( $\mathrm{O}^{\prime}$ Leary et al., 2011). Cognitive function is a complex and highly adaptive neurophysiological complex process that may be affected, both positively and negatively by physiological changes. Increases in the cerebral blood flow, especially in the internal carotid and middle cerebral arteries have been shown to have a positive effect on CP (Ogoh et al., 2014).

Moderate intensity aerobic exercise has been shown to increase arousal levels in the reticular activating system, increasing ascending stimulation of the prefrontal cortex, thus resulting in improved CP (Byun et al., 2014). Exercise has been shown to have an acute positive effect on cognition, with increases in selective attention, processing speed and executive function-related tasks (Alves et al., 2012; Chang et al., 2015b). The range of intensity of aerobic exercise for optimal effects on $\mathrm{CP}$ has been found to be $60-70 \%$ of the maximum heart rate, which is defined as moderate intensity (Arcelin et al., 1997; Chang et al., 2015a; Weng et al., 2015; Yanagisawa et al.,

1 - Department of Physical Therapy New York Institute of Technology, Old Westbury, NY; USA. 
2010). Additionally, high intensity exercise above this optimal range may actually decrease $\mathrm{CP}$ (Rattray and Smee, 2013). There is an inverted Urelationship between the activation level of the central nervous system (CNS) and the intensity of the exercise performed (Kashihara et al., 2009).

A long term training study utilizing video gaming activities has shown that these activities improve executive function, as demonstrated by Maillot et al. (2012). This improvement was dependent on the numerous variables of the gaming activity including task difficulty, motivation, arousal, variability, and feedback. Many studies have speculated that using motivational features such as "exergames", defined as exercise requiring interaction with a game, is beneficial for subjects' exercise compliance. The interaction component of these "exergames" shifts the subject's attention from the physical discomfort to the competition or scenery provided by the game, thus providing a motivational component (Anderson-Hanley et al., 2012a, 2012b). Nouchi et al. (2012, 2013) found in their long term training studies that brain-training games (Brain Age, Nintendo Co., Redmond, WA) significantly improved executive function, working memory, and processing speed; while non-brain-training games had little impact on cognitive performance in separate studies utilizing young and older adults (Nouchi et al., 2012, 2013). Brain-training games have not only been hypothesized to increase cognition by recruiting the prefrontal cortex to complete the task, but also by increasing cerebral hemodynamics (Nouchi et al., 2012, 2013). The above-mentioned studies allude to the potential of beneficial changes in cognitive function from moderate aerobic exercise combined with participating in video games and or exergaming. The mechanism responsible for improved executive function is not fully known, though prior research has proposed improved hemodynamics in the prefrontal cortex as a major contributing factor. Cognitive function is suggested to enhance secondary to improved regional cerebral blood flow and induce an increase in neurotransmitters that facilitate the central nervous system (Kashihara et al., 2009). An increase in the brain-derived neurotrophic factor which participates in neuronal transmission and modulation has also been shown to play a role in improving cognition during acute exercise (Ferris et al., 2007) and chronic exercise (AndersonHanley et al., 2012a).

Long term aerobic exercise and video gaming both appear to provide the physiological stimulus that results in improved executive function. However, there appears to be a lack of knowledge of the acute effects of video gaming on CP. O'Leary et al. (2011) compared the effects of seated rest, seated videogame play, treadmill aerobic exercise, and treadmill-based exergame aerobic exercise at $60 \%$ of the maximal heart rate on $\mathrm{CP}$. Their results demonstrated that single bouts of treadmill exercise improved executive function by increasing the allocation of attentional resources and improved control of interference during cognitively demanding tasks. However, similar benefits were not found following acute bouts of aerobic exergaming or seated videogame participation. The effects of quantity (intensity and duration) of exercise on cognitive performance have been well documented. However, the effect of qualitative mode of exercise as a mediator of cognitive performance remains under-investigated, according to Pesce (2012). The addition of a non-congruent cognitive demanding video game to a specific physical activity may impose a different cognitive demand and effect compared to exergaming. There appears to be a gap in the current knowledge on whether the combination of aerobic exercise while simultaneously gaming leads to greater improvements in $\mathrm{CP}$ than either intervention alone. The purpose of our study was to investigate the effects of video gaming, aerobic exercise (biking), and the combination of these two activities on the domains of $\mathrm{CP}$ : selective attention, processing speed, and executive functioning. We hypothesized that a combination of both aerobic exercise and simultaneous gaming would result in greater $\mathrm{CP}$ than gaming or aerobic exercise, independently.

\section{Methods}

Participants

Forty participants, 16 men and 24 women (mean age $23.7 \pm 1.8$ years) volunteered to participate in this study. Descriptive information of the participants in each of the four groups is presented in Table 1. The participants came from the New York Institute of Technology student 
community. The subjects were selected according to the inclusion/exclusion criteria. Inclusion criteria were as follows: age range: $18-30$ years, good overall health, normal corrected vision and color vision, and that they exercised at least two days/week. The following conditions were considered as exclusion criteria: any cardiopulmonary conditions including asthma within 6 months prior to participation, any major musculoskeletal injuries during the last 6 months, any auditory/vestibular impairment, uncorrected visual problems and any other health issues that would interfere with subject's safety during exercise. Volunteers completed a medical history and health questionnaire in order to screen for the inclusion and exclusion criteria. Each participant's medical history was evaluated for the presence of exclusion criteria by utilizing the Physical Activity Readiness Questionnaire (PAR-Q) and the medical/health questionnaire. All procedures of the investigation were conducted in accordance with the Helsinki Declaration of 1975. The consent form and the study were approved by the Institutional Review Board of the New York Institute of Technology (BHS1115) and the study was registered on www.ClinicalTrail.gov (NCT02437097). All methodological details of this trial were published before the start of data collection.

\section{Design and Procedures}

The study was a 4-arm randomized clinical trial with four conditions and two dependent variables, the Stroop Color and Word test and the Oral Trails B test, which are validated measures of $\mathrm{CP}$. The participants were randomly assigned to one of the four conditions according to a Latin square design: aerobic exercise, video game, combination of aerobic exercise and video game, or the control condition of sitting quietly (Figure 1). The participants were required to attend one initial day and one day of testing. During the initial day, participants were screened for inclusion and exclusion criteria, signed the consent form, and were familiarized with the training and testing protocols. Participants were advised to avoid caffeine, alcohol, heavy exertion, and maintain normal hydration 24 hours prior to testing. Upon signing the consent form, the participants were instructed on how the Stroop Color and Word and Oral Trails B tests would be administered. The participants also familiarized themselves with the Brain Age video game (Nintendo Co., Redmond, WA) prior to testing via a trial run of 3 minutes. The second session consisted of pre- and post-testing of $\mathrm{CP}$ in one of the four conditions.

Cognitive Tasks: Stroop Color and Word and Oral Trails B

Cognitive performance was assessed by the Stroop Color and Word test (Golden and Freshwater, 2002). The Stroop test has been frequently utilized to assess the effects of acute exercise on cognitive performance (Barella et al., 2010; Byun et al., 2014; Tam, 2013; Yanagisawa et al., 2010). The Stroop test is a timed performance task that consists of three sections: the Word section contains the names of three colors (blue, red, green) in black font; the Color section contains rows of the letter " $\mathrm{X}$ " with each " $\mathrm{X}$ " in one of the three different colors (blue, red, green); and the Color-Word section contains the words from the Word section, but with the font of each word in a color that conflicts with the color that the word describes. Subjects were instructed to read the words or name the font colors as quickly as possible for 45 seconds in each of the three subsections. The Color-Word section presents the two conflicting sources of color information causing a competing effect known as Stroop interference. A conflict arises between reflexively reading the word's description and naming the color of the font. The individual must choose one response by suppressing the other choice (response inhibition). The Word and Color Tscores are assessments of selective attention and processing speed, while the Color-Word T-scores assess response inhibition which is an index of executive function, specifically inhibition and impulsivity (Barella et al., 2010). T-scores reflect the raw scores adjusted for a person's age and education level, higher T-scores represent greater CP (Golden and Freshwater, 2002). The Oral Trails $\mathrm{B}$ test was also used to measure executive function. In this test the participant draw a line to connect circled numbers and letters in an alternating numeric and alphabetic sequence as rapidly and accurately as possible while being timed. Less time elapsed represented greater cognitive performance. The Oral Trails B test provides information about visual search speed, attention, scanning, speed of processing, abstraction, mental flexibility, and the ability to 
maintain two trains of thought simultaneously and rapidly shift attention between the two, which is another measure of executive function (Arnett et al., 1995; Salthouse, 2011). Both tests required approximately 5 minutes to administer. The Stroop and the Oral Trails B tests have both been used extensively in the literature to measure changes in executive function post-exercise (Alves et al. 2012; Anderson-Hanley et al., 2012a, 2012b; Byun et al., 2014; Chang et al., 2015a;Mekari et al., 2015; Tam, 2013). Assessment of CP, using the Stroop Color and Word and Oral Trails B tests occurred before and after completion of one of the four thirty-minute conditions: 1) Aerobic exercise only 2) Aerobic exercise and Gaming simultaneously 3) Gaming only 4) Control condition of sitting quietly.

Aerobic Exercise Condition

Participants performed 30 minutes of cycling at an intensity of 60 to $70 \%$ of the maximum heart rate based on their predicted maximum heart rate of 220 minus their age. The investigators monitored the subject's heart rate by utilizing a Polar heart rate monitor (Polar Electro Inc., Lake Success, NY) to maintain their heart rate in the specified range. The aerobic cycling exercise was performed on a Monarch 818E Lower Body Ergometer (Monark Exercise, Stockholm, Sweden).

Video Gaming Condition

Participants in the video game group played Brain Age: Train Your Brain in Minutes a Day! (Nintendo Co., Redmond, WA) on a Nintendo 3DS for 30 minutes in a seated position. We used the calculation X 100 game, where participants were required to answer a total of a 100 questions including addition, subtraction, and multiplication. Participants were on a continuous loop of questions for thirty minutes. The Brain Age game was chosen owing to its proposed beneficial effects on cognitive performance (Nouchi et al., 2012, 2013).

Combined Aerobic Exercise and Video Gaming Condition

Participants cycled on a Monarch 818E Lower Body Ergometer (Monark Exercise, Stockholm, Sweden) while playing Brain Age: Train Your Brain in Minutes a Day! (Nintendo Co., Redmond, WA) on a Nintendo 3DS. Participants performed 30 minutes of cycling at the same intensity of the aerobic exercise condition, 60 to
$70 \%$ of the maximum heart rate based on their predicted maximum heart rate of 220 minus their age. The investigators monitored the subject's heart rate by utilizing a Polar heart rate monitor (Polar Electro Inc., Lake Success, NY) to maintain their heart rate in the specified range. The game was placed on a rolling table in front of the cycle so that the subject was able to cycle and play the game simultaneously.

\section{Control Condition}

Participants were instructed to sit quietly for 30 minutes with their eyes open in an isolated, quiet room.

Statistical Analyses

Statistical analyses were performed utilizing SPSS for Windows (version 22.0, Chicago, Ill.), using a mixed design. In order to test the hypothesis, we performed a $2 \times 4$ mixed ANOVA for each Stroop Color and Word T-score and Oral Trails B test score, with time (pre and post condition) and the four conditions as the main effects. The assumption of sphericity was tested using the Mauchly's test. In the event that sphericity was violated a Greenhouse-Geisser correction factor was applied. A priori sample size calculations revealed that 40 subjects were required to detect observable differences at power of $80 \%$. In the event of significant interaction and/or main effects, post hoc pairwise comparisons were performed using single-degree of freedom contrasts with a Bonferroni correction. An alpha level of $p<0.05$ was used for all statistical comparisons.

\section{Results}

The physiological characteristics of the groups are presented in Table 1. The groups were not significantly different for any of the characteristics according to a one-way ANOVA. The means and standard deviations of the four Stroop Color and Word T-scores and Oral Trials B scores, pre- and post-condition are presented in Table 2. The results of the 2x4 mixed ANOVA for the Stroop Color and Word and Oral Trials B tests are presented in Table 3. The interaction effect (interaction of time $x$ condition) and main effect of condition were not significant for either the Stroop Color and Word or Oral Trials B (Table 3). The main effect of time was significant for Word, $F_{1,36}=51.58, p=0.001$, Color, $F_{1,36}=21.27, p=0.001$ and Color-Word, $F_{1,36}=13.68, p=0.001$. The main 
effect of time was also significant for the Oral Trail B test, $F_{1,36}=4.40, p=0.043$. In order to test the hypothesis that a combination of both aerobic exercise and simultaneous gaming would result in greater $\mathrm{CP}$ than gaming or aerobic exercise alone, a priori pairwise comparisons of the simple effects were conducted when there was a significant main effect for time (pre to post) without a significant interaction effect. According to Tybout et al. (2001), it is appropriate to test for simple effects when an a priori expectation exists even though the interaction term fails to achieve significance. Post hoc comparisons within each condition utilizing paired t-tests with Bonferroni correction are presented in Table 4. In the Bike condition there were significant improvements from pre to post-test in the Stroop Word (selective attention and processing speed), $p=0.004$, Color (selective attention and processing speed), $p=$ 0.020, and Stroop Color-Word (executive function), $p=0.001$. Significant improvements in the Stroop Word were noted in the Game $(p=$ $0.012)$ and combined Bike and Game $(p=0.008)$ conditions. Although the main effect of time was significant for the Oral Trail B test, post hoc comparisons for each condition were not significant.

\begin{tabular}{|c|c|c|c|c|}
\hline \multicolumn{5}{|c|}{ Characteristics of participants in each group } \\
\hline & Control & Bike & Game & Bike \& Game \\
\hline Age (years) & $23.7 \pm 1.9$ & $23.1 \pm 2.1$ & $24.2 \pm 2.3$ & $23.9 \pm 1.0$ \\
\hline Body Height $(\mathrm{cm})$ & $171.5 \pm 7.1$ & $175.3 \pm 9.3$ & $170.7 \pm 13.2$ & $169.5 \pm 8.5$ \\
\hline Body Mass (kg) & $73.0 \pm 7.5$ & $69.8 \pm 9.7$ & $68.2 \pm 8.5$ & $71.6 \pm 15.2$ \\
\hline & Values a & I me ment & ard deviation & \\
\hline
\end{tabular}

\begin{tabular}{|c|c|c|c|c|}
\hline & & & \multicolumn{2}{|c|}{ Table 2} \\
\hline & Word & Color & Color-Word & Oral Trails B \\
\hline Pre Control & $52.2 \pm 11.0$ & $52.7 \pm 10.3$ & $54.3 \pm 11.2$ & $44.4 \pm 12.2$ \\
\hline Post Control & $55.2 \pm 12.5$ & $54.6 \pm 12.3$ & $57.1 \pm 10.2$ & $50.2 \pm 12.0$ \\
\hline Pre Bike & $48.0 \pm 7.7$ & $49.7 \pm 8.7$ & $51.5 \pm 8.4$ & $52.3 \pm 13.6$ \\
\hline Post Bike & $54.2 \pm 10.8$ & $55.8 \pm 10.8$ & $58.3 \pm 9.4$ & $49.8 \pm 17.9$ \\
\hline Pre Game & $50.5 \pm 10.1$ & $50.7 \pm 8.4$ & $55.0 \pm 9.1$ & $46.0 \pm 14.5$ \\
\hline Post Game & $55.2 \pm 11.2$ & $53.0 \pm 9.7$ & $58.1 \pm 10.1$ & $50.8 \pm 13.2$ \\
\hline Pre Bike \& Game & $49.7 \pm 3.8$ & $52.7 \pm 8.2$ & $54.8 \pm 10.0$ & $50.6 \pm 15.1$ \\
\hline Post Bike \& Game & $56.2 \pm 6.1$ & $56.1 \pm 8.7$ & $57.1 \pm 8.8$ & $57.1 \pm 7.1$ \\
\hline \multicolumn{5}{|c|}{$\begin{array}{c}\text { Values are given as mean } \pm \text { standard deviation } \\
\text { Stroop Scores are presented as T-scores } \\
\text { Oral Trial B Scores are in seconds }\end{array}$} \\
\hline
\end{tabular}


Table 3

Results of $2 \times 4$ Mixed ANOVA for Main and Interaction Effects

\begin{tabular}{lll}
\hline Dependent Variable & Main Effect of & Main Effect of \\
Time & Condition & $\begin{array}{l}\text { Interaction Effect } \\
\text { (Time x Condition) }\end{array}$
\end{tabular}

\begin{tabular}{lccc}
\hline Word & $p=0.001+$ & $p=0.935$ & $p=0.296$ \\
Color & $p=0.001+$ & $p=0.938$ & $p=0.201$ \\
Color-Word & $p=0.001+$ & $p=0.982$ & $p=0.390$ \\
Oral Trails B & $p=0.043^{*}$ & $p=0.645$ & $p=0.250$
\end{tabular}

${ }^{*}$ Denotes a significant difference between pre and post test scores at $p<0.05$

+ Denotes a significant difference between pre and post test scores at $p<0.01$

Table 4

Post Hoc Comparisons for Main Effect of Time with Paired $t$-tests corrected with Bonferroni

\begin{tabular}{lllll}
\hline Pre to Post & Bike & Game & Control & Bike \& Game \\
& & & & \\
\hline Word & $p=0.004+$ & $p=0.012^{*}$ & $p=0.421$ & $p=0.008+$ \\
Color & $p=0.020^{*}$ & $p=0.436$ & $p=0.448$ & $p=0.368$ \\
Color-Word & $p=0.001+$ & $p=0.900$ & $p=0.576$ & $p=0.900$ \\
& & $p=0.315$ & $p=0.200$ & $p=0.400$ \\
Oral Trails B & $p=0.424$ & & & \\
& & & &
\end{tabular}

${ }^{*}$ Denotes a significant difference between pre and post test scores at $p<0.05$

+ Denotes a significant difference between pre and post test scores at $p<0.01$ 
Figure 1-Study Flow Diagram

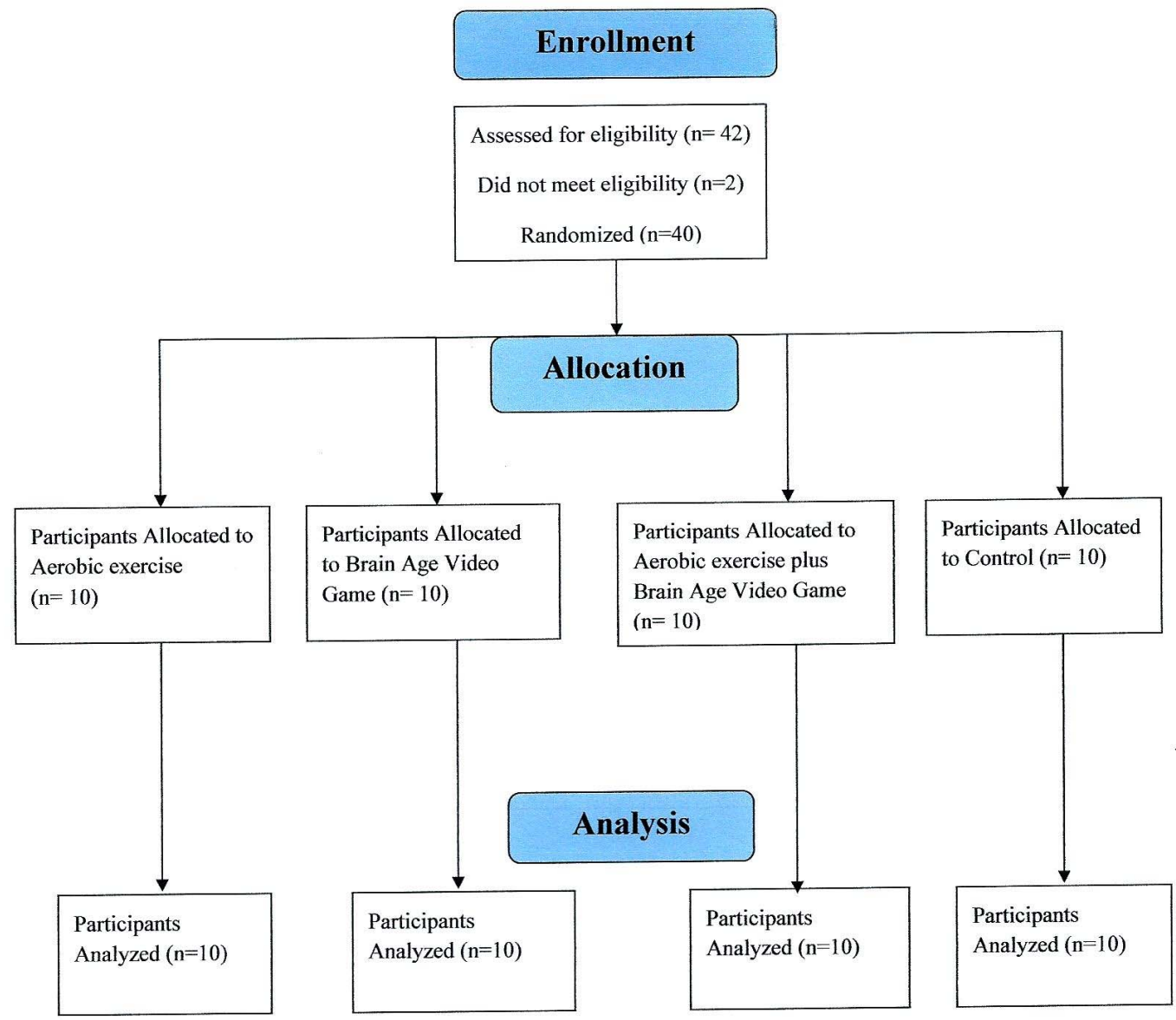

\section{Discussion}

In the present study we attempted to determine the cognitive effects of participating in a video game, aerobic exercise, and a combination of both gaming and aerobic exercise, in the areas of selective attention, processing speed, and executive functioning. The current study demonstrated positive acute effects of a bout of moderate intensity aerobic exercise on improving selective attention, processing speed and executive function. Our results compare favorably to previous studies investigating the acute effects of exercise on cognitive performance (Alves et al., 2012; Chang et al., 2012a; O'Leary et al., 2011). Alves et al. (2012) demonstrated that acute bouts of treadmill-based aerobic exercise improved executive function as measured by the Stroop Color and Word test in middle-aged women. They also did not find any significant improvements in the Oral Trails B test following exercise, similarly to our findings. Chang et al. (2015a) demonstrated a dose-dependent response of moderate intensity aerobic exercise on cognitive performance. Their findings showed that a 20-minute bout of moderate intensity cycle ergometry had positive effects on cognitive performance, as verified by a shorter response time and greater accuracy in the Stroop Color and Word test when compared to 10 and $45 \mathrm{~min}$ of exercise. Our results compare favorably to O'Leary et al. (2011) who also found improvements in executive function following treadmill-based aerobic exercise for $20 \mathrm{~min}$, but 
not after exergaming or videogaming alone. In our study, all groups except the control group displayed significant improvements in selective attention and processing speed, however only the aerobic exercise group improved in the higher cognitive process of executive function, as measured by the Stroop Color and Word test.

Improved hemodynamics plays an important role in enhancing cognitive function. Research supports that moderate-intensity exercise increases a blood flow to the CNS, specifically to the reticular activating system, which is responsible for arousal, and to the prefrontal cortex, which is responsible for the majority of executive function (Byun et al., 2014; Giles et al., 2014; Mekari et al., 2015). The improved cerebral blood flow, resulting from moderate-intensity exercise may also increase cognitive performance by providing additional energy substrates, e.g., glucose and oxygen for improved neurological functioning (Kashihara et al., 2009). Mekari et al. (2015) found significant differences in oxygenated hemoglobin and associated executive function when comparing high, moderate, and low intensity aerobic exercise. They found that higher intensities $(85 \%$ peak power output) caused a significant decrease in cerebral oxygenated hemoglobin which was associated with impaired executive function. Kashihara et al. (2009) proposed that moderate intensity exercise improved cognition, while exercise at higher intensities, defined as exercise at or above the lactate threshold, attenuated cognitive function in an inverted U-relationship.

Our Game condition and the combined

Bike \& Game condition demonstrated improvements in selective attention and processing speed, but not in executive function. The Control condition did not demonstrate improvements in any of the measured domains of cognitive performance. Nouchi et al. (2012; 2013) showed significant improvements in executive function, working memory and processing speed after playing the Brain Age (Nintendo Co., Redmond, WA) for 15 minutes, five days per week, for four weeks in two separate studies utilizing young adults and older adults in separate studies. A long-term (3 months) training study in older adults by Anderson-Hanley et al. (2012b) showed significant improvement in executive function while "exergaming" on a cycle compared to traditional cycling using a recumbent stationary bike. They proposed that the primary reason for the improvement was an increase in the brain-derived neurotrophic factor in the cybercycling group (Anderson-Hanley et al., 2012a). They utilized "exergaming" in their research and their effects may be different from the combination of exercise and video gaming because the goals of the game and exercise in "exergaming" are aligned. Also, we are not able to compare our results with Nouchi et al. (2012; 2013) and Anderson-Hanley et al. (2012a; 2012b) because the acute effects of exercise and or gaming may differ from the chronic effects on executive function. Also, different game genres have positive effects on different cognitive skills according to Oei and Patterson (2013). The Brain Age game (Nintendo Co., Redmond, WA) that we used in this study was expected to improve attention, processing speed, and executive functioning according to Nouchi et al. (2012; 2013). O'Leary et al. (2011) also did not find acute improvements in executive function following a bout of "exergaming" or video gaming. Their results support our findings of the acute effects of gaming on attention and processing speed, but not on executive function. Our results support previous findings and provide evidence that a bout of aerobic exercise with simultaneous gaming has positive acute effects on attention and processing speed for young adults, but no beneficial effect on executive function. The long term or cumulative effects of video gaming differ from the acute effects on improving executive function.

Our hypothesis that the combination of biking and gaming would elicit the greatest acute improvement in cognitive performance was not supported. Despite the complexity and inherent processing speed of the human brain, it is limited by attentional capacity, according to Marois and Ivanoff (2005) and McDowd (2007). Attention can be allocated to multiple processing tasks as long as the allocation does not exceed the amount of available resources (McDowd, 2007). If tasks approach the limits of available attentional capacity, a performance decrement will occur, therefore it is possible that increased physical and mental cognitive demands, beyond a certain threshold can increase the probability for cognitive errors (Marois and Ivanoff, 2005; 
McDowd, 2007). This decline in performance is referred to as the dual-task deficit. This dual-task deficit may explain our findings of a lack of improvement in executive function in the combined Bike \& Game condition. The combined tasks of stationary biking and gaming may have surpassed the limit of attentional capacity, which may have ultimately led to an increase in errors during the Stroop Color and Word and Oral Trials B tests.

Our current study and previous studies support that moderate-intensity aerobic exercise can acutely improve executive function (Alves et al., 2012; Chang et al., 2015a; O'Leary et al., 2011; Tam, 2013). The external validity of our study is limited to young college-aged adults and should not be extrapolated to other age groups. The subjects in the Nouchi et al.'s (2012; 2013) studies participated in only 15 minutes of gaming versus 30 minutes of gaming in our study. The optimal time for gaming to acutely improve CP must also be investigated and established. Another limitation is that the outcome measures used to assess $\mathrm{CP}$ in this study might not have captured the changes in all of the various domains of $\mathrm{CP}$, specifically executive function. Thus, to be more accurate when comparing the effects of gaming and exercising on $\mathrm{CP}$, standardized cognitive batteries, which assess the primary areas of $\mathrm{CP}$, should be developed for future studies. Future research which focuses on the acute effects of different types and duration of exercise, gaming, and combined exercise and gaming on $\mathrm{CP}$ is warranted.

In conclusion, moderate intensity, stationary cycling of 30-minutes duration resulted in improved processing speed, selective attention, and executive function. However, combined cycling and gaming or gaming alone also increased $\mathrm{CP}$, but only in the areas of selective attention and processing speed. Combined biking and gaming may surpass the limits of attentional capacity, thereby increasing errors in the area of executive function. Performing 30 minutes of moderate intensity aerobic exercise immediately prior to mentally challenging tasks or as part of a warm up prior to athletic competition may enhance $\mathrm{CP}$ in the areas of attention and processing speed, as well as improve decisionmaking in college-aged adults and athletes. Our results suggest that the fatiguing effects of a combined physically and mentally challenging task that extends after the exercise cessation may overcome the eventual beneficial cognitive effects derived from the physical exercise.

\section{Acknowledgements}

We would like to thank all the subjects for participating in the study.

\section{References}

Alves CR, Gualano B, Takao PP, Avakian P, Fernandes RM, Morine D, Takito MY. Effects of acute physical exercise on executive functions: a comparison between aerobic and strength exercise. J Sport Exerc Psychol, 2012; 34(4): 539-549

Anderson-Hanley C, Arciero PJ, Brickman AM, Nimon JP, Okuma N, Westen SC, Merz ME, Pence BD, Woods JA, Kramer AF, Zimmerman EA. Exergaming and older adult cognition: a cluster randomized clinical trial. Am J Prev Med, 2012a; 42(2): 109-119

Anderson-Hanley C, Arciero PJ, Westen SC, Nimon J, Zimmerman E. Neuropsychological benefits of stationary bike exercise and a cybercycle exergame for older adults with diabetes: an exploratory analysis. J Diabetes Sci Technol, 2012b; 6(4): 849-857

Arcelin R, Brisswalter J, Delignierres D. Effects of physical exercise duration on decision- making performance. J Hum Movement Stud, 1997; 32: 123-140

Arnett JA, Labovitz SS. Effect of physical layout in performance of the Trail Making Test. Psychol Assessment, 1995; 7(2): 220-221

Barella LA., Etnier JL, Chang Y K. The immediate and delayed effects of an acute bout of exercise on 
cognitive performance of healthy older adults. J Aging Phys Act, 2010; 18: 87-98

Byun K, Hyodo K, Suwabe K, Ochi G, Sakairi Y, Kato M, Dan I, Soya H.. Positive effect of acute mild exercise on executive function via arousal-related prefrontal activations: an fNIRS study. Neuroimage, 2014; 98: 336-345

Chang YK, Chu CH, Wang CC, Wang YC, Song TF, Tsai CL, Etnier JL. Dose-response relation between exercise duration and cognition. Med Sci Sports Exerc, 2015a; 47(1): 159-165

Chang YK, Chu CH, Wang CC, Song TF, Wei GX. Effect of acute exercise and cardiovascular fitness on cognitive function: an event-related cortical desynchronization study. Psychophysiology,2015b; 52(3): $342-351$

Ferris LT, Williams JS, Shen CL. The effect of acute exercise on serum brain-derived neurotrophic factor levels and cognitive function. Med Sci Sports, 2007; 39(4): 728-734

Giles GE, Brunyé TT, Eddy MD, Mahoney CR, Gagnon SA, Taylor HA, Kanarek RB. Acute exercise increases oxygenated and deoxygenated hemoglobin in the prefrontal cortex. Neuroreport, 2014; 25(16): 1320-1325

Golden C, Freshwater S. A manual for the adult stroop color and word test. Chicago IL: Stoelting, 1-62; 2002

Kashihara K, Maruyama T, Murota M, Nakahara Y. Positive effects of acute and moderate physical exercise on cognitive function. J Physiol Anthropol, 2009; 28(4): 155-64

Maillot P, Perrot A, Hartley A. Effects of interactive physical-activity video-game training on physical and cognitive function in older adults. Psychol Aging, 2012; 27(3): 589-600

Marois R, Ivanoff J. Capacity limits of information processing in the brain. Trends Cogn Sci, 2005; 9(6): 296305

McDowd JM. An overview of attention: behavior and brain. J Neurol Phys Ther, 2007; 31(3): 98-103

Mekari S, Fraser S, Bosquet L, Bonnéry C, Labelle V, Pouliot P, Lesage F, Bherer L. The relationship between exercise intensity, cerebral oxygenation and cognitive performance in young adults.Eur J Appl Physiol, 2015; 115(10): 2189-2197

Nouchi R, Taki Y, Takeuchi H, Hashizume H, Nozawa T, Kambara T, Sekiguchi A, Miyauchi CM, Kotozaki $\mathrm{Y}$, Nouchi $\mathrm{H}$, Kawashima R. Brain training game boosts executive functions, working memory and processing speed in the young adults: a randomized controlled trial. PLoS ONE, 2013; 8(2):e55518

Nouchi R, Taki Y, Takeuchi H, Hashizume H, Akitsuki Y, Shigemune Y, Sekiguchi A, Kotozaki Y, Tsukiura $\mathrm{T}$, Yomogida $\mathrm{Y}$, Kawashima R. Brain training game improves executive functions and processing speed in the elderly: a randomized controlled trial. PLoS ONE, 201 7(1): e29676

Oei AC, Patterson MD. Enhancing cognition with video games: a multiple game training study. PLoS ONE, 2013; 8(3): e58546

Ogoh S, Tsukamoto H, Hirasawa A, Hasegawa H, Hirose N, Hashimoto T. The effect of changes in cerebral blood flow on cognitive function during exercise. Physiol Rep, 2014; 2(9): 1-8.

O'Leary KC, Pontifex MB, Scudder MR, Brown ML, Hillman CH. The effects of single bouts of aerobic exercise, exergaming, and videogame play on cognitive control. Clin Neurophysiol, 2011; 122(8): 15181525

Pesce C. Shifting the focus from quantitative to qualitative exercise characteristics in exercise and cognitive research. J Sport Exerc Psychol, 2012; 34: 766-786

Rattray B, Smee D. Exercise improves reaction time without compromising accuracy in a novel easy-toadminister tablet-based cognitive task. J Sci Med Sport, 2013; 16(6):567-570

Salthouse TA. What cognitive abilities are involved in trail-making performance? Intelligence, 2011; 39(4): $222-232$

Tam ND. Improvement of processing speed in executive function immediately following an increase in cardiovascular activity. Cardiovas Psychiatry Neurol, 2013; 1-6 
Tybout A, Sternthal B, Keppel G, Analysis of variance. J Consum Psychol, 2001; 10(1-2): 5-35

Weng TB, Pierce GL, Darling WG, Voss MW. Differential effects of acute exercise on distinct aspects of executive function. Med Sci Sports Exerc, 2015; 47(7):1460-1469

Yanagisawa H, Dan I, Tsuzuki D, Kato M, Okamoto M, Kyutoku Y, Soya H. Acute moderate exercise elicits increased dorsolateral prefrontal activation and improves cognitive performance with Stroop test. Neuroimage, 2010; 50(4): 1702-1710

\section{Corresponding author:}

\section{Peter C Douris PT, DPT, EdD, OCS}

Professor of Physical Therapy, Department of Physical Therapy

New York Institute of Technology, Old Westbury, NY 11568

Phone516 686-7688; Fax: 516 686-7699

E-mail: pdouris@nyit.edu 